\title{
Face à la mort : la communication avec l'au-delà chez les Oudmourtes dans le contexte des représentations d'avant-mort
}

In Face of Death: Communicational Behaviour of the Udmurt in the Context of Pre-Death Representations

Silmitsi surmaga: udmurtide suhtluskäitumine surmaeelsete kujutluste

kontekstis

ПРЕД ЛИКОМ СМЕРТИ: КОММУНИКАТИВНОЕ ПОВЕДЕНИЕ УДМУРТОВ В

КОНТЕКСТЕ ОКОЛОСМЕРТНЫХ ПРЕДСТАВЛЕНИЙ

\section{Nikolaj Anisimov}

Traducteur : Aleksi Moine

\section{CpenEdition}

\section{Journals}

Édition électronique

URL : https://journals.openedition.org/efo/7224

DOI : $10.4000 /$ efo. 7224

ISSN : 2275-1947

Éditeur

INALCO

Édition imprimée

ISBN : 978-2-343-12463-6

ISSN : 0071-2051

\section{Référence électronique}

Nikolaj Anisimov, «Face à la mort : la communication avec l'au-delà chez les Oudmourtes dans le contexte des représentations d'avant-mort », Études finno-ougriennes [En ligne], 48| 2017, mis en ligne le 20 juillet 2017, consulté le 08 juillet 2021. URL : http://journals.openedition.org/efo/7224 ; DOI https://doi.org/10.4000/efo.7224

Ce document a été généré automatiquement le 8 juillet 2021.

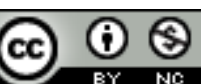

Études finno-ougriennes est mis à disposition selon les termes de la Licence Creative Commons Attribution - Pas d'Utilisation Commerciale 4.0 International. 


\title{
Face à la mort : la communication avec l'au-delà chez les Oudmourtes dans le contexte des représentations d'avant-mort
}

\author{
In Face of Death: Communicational Behaviour of the Udmurt in the Context of \\ Pre-Death Representations \\ Silmitsi surmaga: udmurtide suhtluskäitumine surmaeelsete kujutluste \\ kontekstis
}

ПРЕД ЛИКОМ СМЕРТИ: КОММУНИКАТИВНОЕ ПОВЕДЕНИЕ УДМУРТОВ В

КОНТЕКСТЕ ОКОЛОСМЕРТНЫХ ПРЕДСТАВЛЕНИЙ

Nikolaj Anisimov

Traduction : Aleksi Moine

Dans la religion de nombreux peuples du monde, la mort ne se comprend pas comme la fin de l'existence d'un individu, mais plutôt comme le début de son voyage vers une autre vie. Ainsi, selon les conceptions religieuses des Oudmourtes, la mort est une des étapes de la transition vers le monde des morts, vers la foule des ancêtres. Dans les chants populaires oudmourtes, l'être humain «ne paraît que comme invité temporaire » dans ce monde. Lorsqu'ils atteignent un âge avancé, les Oudmourtes décident de leur vêtement funéraire et se préparent moralement à leur propre mort. À propos de la transition vers le monde des morts, on utilise l'expression :

СОПАЛ ДУННЕЕ КОШКИЗ, ТАРЕ КУЛЭМЪЁСЛЭН ГУРТАЗЫ УЛЭ

(littéralement : Il est parti dans «le monde de l'autre côté ${ }^{1}$ » il vit maintenant dans

le village des morts ${ }^{2}$.

La période qui précède la mort est, pour l'individu, une période critique : c'est un moment où il entre en participation particulièrement étroite avec le monde des morts. Les expériences d'avant-mort vécues à cette période comportent habituellement une multiplicité de dimensions - physiques, psychologiques et surnaturelles. Une analyse 
des matériaux obtenus de manière empirique et des informations tirées de publications scientifiques permet de distinguer les phénomènes suivants, caractéristiques de cette période :

- les signes symboliques d'un décès proche ;

- les souffrances physiques et les changements externes du mourant;

- la rencontre et le dialogue avec des défunts membres de la famille;

- la rencontre avec un être du monde des morts ;

- la conscience ou l'inconscience de sa propre mort ;

- les cas de syncope et de renaissance.

\section{Les signes de la mort}

D'après nos informateurs, une personne ressent, avant sa mort, une forme d'inquiétude intérieure ; parfois, lorsqu'elle prend conscience de sa propre mort, c'est de l'effroi, de la panique. Avant son départ vers l'autre monde, l'individu est confronté à des phénomènes surnaturels, populairement interprétés comme des signes de l'approche du décès. Ces signes peuvent être :

- un comportement non naturel des animaux et des oiseaux (par exemple, un oiseau qui s'envole dans la maison ou se cogne contre la fenêtre) :

УКНО ПИЯЛАЕ ЛОБАСЬ ТЫЛОБУРДО МОГӞЕ. КЫШНОПАЛА КЕ - КЫШНОМУРТЛЫ ЛУЭ. МОГӞЕ КЕ, ИВОР КЫЛӤСЬКЕ НИ, ВЫЖЫ-КУМЫЛЭН. ТӦР ШОРЫ КЕ МОГӞЕ ПИОСМУРТЛЭН КЫЛӤСЬКЕ КУЛЭМ ИВОРЕЗ.

(TTA : district Dubrovskij, raïon de Kijasovo, RO, 2010)

Un oiseau se cogne contre la vitre d'une fenêtre. Si c'est du côté de la cuisine, [la mort] va arriver à une femme. Si [l'oiseau] se cogne [contre la fenêtre], on va bientôt entendre la nouvelle [d'une mort] dans la famille. Si cela arrive du côté du coin rouge ${ }^{3}$, ce sera la nouvelle du décès d'un homme ${ }^{4}$;

- de « mauvais » rêves ;

- une rencontre avec des êtres du monde de l'autre côté (par exemple, les âmes des morts, des esprits de la forêt ou de l'eau);

- le flétrissement et la mort d'arbres qui ont été plantés, de plantes, etc. :

УЙВӦТТЯ КЕ, ЛЫСО ПИСПУ КЕ - ПИОСМУРТЛЫ ЛУЭ. УЙВӦТЫН ПОГРАМ ЛЫСО ПИСПУЭЗ АӞӞИСЬКОД КЕ, ПИОСМУРТЛЫ ЛУЭ. КУАРО ПИСПУ КЕ ЛУЭ КЫШНОМУРТЛЫ ЛУЭ. НюЛЭС ПОГЫРЪЯСА КЕ АӞӞИД, МАТЫСь ВЫЖЫ-КУМЫЛЫ ЛУЭ. [...] ПАЛЛЯН ПЫДПЫДэС КЕ ЛЫДэ, КУЛэм КылӥськОз. [...] тодэмЛЫ ЛУэ со.

(TTA : district Dubrovskij, raïon de Kijasovo, RO, 2010)

Si l'on voit en rêve un conifère, [la mort] va arriver à un homme. Si tu vois en rêve un conifère qui a été coupé, [la mort] va arriver à un homme. Si tu vois un arbre avec des feuilles caduques, c'est à une femme que [la mort] va arriver. Si dans un rêve tu abats une forêt, [la mort] va survenir à un membre proche de ta famille. [...] Si le pied gauche te démange, tu vas recevoir des nouvelles d'un décès. [...] Cela va arriver à [quelqu'un] que tu connais.

Voir en rêve du bois abattu/endommagé est considéré comme un mauvais présage dans d'autres cultures également. Chez les Khakasses, par exemple, « voir en rêve un mélèze, [...] annonce une mort, surtout la mort d'un homme » (Kustova, 2009, p. 50).

Pour prévenir une mort non désirée, les Oudmourtes accomplissent une série d'actions qui comportent aussi des éléments verbaux. Ainsi, lorsqu'une poule se met à chanter comme un coq, on lui coupe la tête en lui adressant ces mots :

эн МЫДлАНьяськЫ! АслЫд МЕД лУОз!

(TTA : district Dubrovskij, raïon de Kijasovo, RO, 2014) 
Ne t'entête pas (littéralement : n'agis pas à l'envers)! Que ce soit sur toi que vienne [la mort]!

Lorsqu'un chat défèque devant quelqu'un, ou s'attaque à un chien qui a la tête baissée, on crache en réponse, et on dit:

АСЛАД ЙЫРАД МЕД ЛУОЗ!

(TTA : district Dubrovskij, raïon de Kijasovo, RO, 2010)

Que ce soit sur ta tête que vienne [la mort] !

Dans d'autres situations non standard/non naturelles, on prononce une formule verbale universelle :

ПЕРЕСЬ МУРТЛЫ/ПЕРЕСЬЁСЛЫ МЕД ЛУОЗ.

Que [la mort] advienne à une personne âgée/aux vieillards.

Les Oudmourtes accordent une signification particulière aux rêves dans lesquels on voit la mort d'un individu, son enterrement et/ou sa tombe. Selon les représentations populaires des Oudmourtes, ces rêves sont un bon signe: la personne a encore une longue vie à vivre. Dans la culture des Khakasses, des songes similaires ne sont pas non plus considérés comme dangereux pour les vivants (Kustova, 2009, p. 53). C'est en revanche un mauvais signe de se voir soi-même en rêve, ou de voir l'un de ses parents, pieds nus et errant à travers la terre. Dans ce contexte précis, la sémantique négative provient, probablement, de ce que le pied dénudé, dans les rituels populaires, sert d'indicateur du monde inférieur (de l'autre monde), dans lequel on ne peut pénétrer que pieds nus (Mazalova, 2001, p. 42 ; Semenov, 1992, p. 113). De même, on juge que la mort est proche lorsqu'on voit fréquemment des rêves dans lesquels des parents ou des connaissances décédées "appellent " à venir les accompagner dans l'autre monde. D'après les récits des informateurs, les vivants sont le plus souvent dérangés par les âmes de ceux qui sont morts d'une mort non naturelle. Selon les représentations populaires, ces derniers peuvent agir sur le terme de la vie des vivants : envoyer des maladies, des malheurs, une mort prématurée. Dans ce genre de situations, les Oudmourtes, afin de se débarrasser du défunt importun et de se soustraire à la mort, accomplissent un rituel mémoriel spécial, сьӦРЛАНЬ/БЕРЛАНЬ КУЯСьКОН (littéralement : le lancement derrière la limite/en arrière) : au cimetière même ou bien le dos tourné vers l'ouest ou vers le cimetière, ils jettent un morceau de nourriture par-dessus leur épaule gauche et ils adressent des reproches à voix haute au défunt. Nous présentons ci-dessous quelques récits de cette cérémonie, que nous avons réussi à noter lors de nos expéditions :

ОДНОКЛАССНИКСЭ КАЛЫЗ БЫРИЗ ВӦТАСА. ПИЧИ ДЫРЪЯЗ КЫШНО НО БАСЬТО ШУЭ ВАЛ. И ВЕСЬ-ВЕСЬ ВӦТАМИСЬТЫЗ ОЗЬЫ, ТУКЛЯЧИ, ПЕ, «ПАЛЛЯН ПЕЛЬПУМТӤД КУЯСЬКЫ АЙ, КЕН», ПЕ, ШУЭ. «СОБЕРЕ ДУГДӤ, МАМА», - ШУЭ. [...] КОНЕШНО, ШАЙ УЛЫН БЕРЕ, УЛЛАНЬ КУЯСЬКИЗ НИ.

(TTA : district Staraja Sal'ja, raïon de Kijasovo, RO, 2014)

[La fille de notre informatrice] était fatiguée de toujours voir en rêve un élève de la première classe. Dans son enfance, il disait même, il la prendrait pour épouse. Et parce qu'elle rêvait sans cesse de lui, sa belle-mère lui a dit : "Accomplis le rituel [littéralement : le lancement/le don] par-dessus ton épaule gauche, ma belle-fille/ ma bru ». "Après, maman a arrêté [de le voir en rêve] ", elle dit. [...] Bien sûr, comme le cimetière est dans le bas de la rivière, c'est vers le bas de la rivière qu'elle l'a accompli [littéralement : le lancement/le don en arrière].

КУЛИКОВ А.Н.: «ТАНИ МЫНАМ ЭШЕ ВАЛ ТУЖ УМОЙ. СО АССЭ АЧИЗ БЫДТЙЗ. ТАЕ НО СЬӦРАЗ ӦТЕ ВАЛ. И ВОТ ТЫКАК КАРИЗ, „ОЙДО” ШУСА. СЫРЕ СЬӦРЛАНЬ КУЯСЬКИ БОЛЬШЕ ӦЙ вӦТА.» 
КУЛИКОВА З.Ф.: “ ТЫШКАСЬКЫСА, ПЕ, КУЯСЬКОНО: „КУЛӤД КЕ, КЫЛЛЬЫ!” МАТЕРИТЬТЫСА, ТЫШКАСЬКЫСА, СЬӦРЛАНЬ КУЯСЬКИСЬКОД. „МОН ТОНЭ НУСАЛ НО, АРКАШЕД ВАНЬ АЙ”, - ШУИЗ. [...] ПРОСТО ШАЙВЫЛЫН, ТАЗЬЫ СЬӦРАМ КУЯЙ: „ЭШ ШЕДЬТЫ ОТЫН! МЫНАМ ТА ДУННЕЫН УЖЕ ТРОС АЛИ!” ШУСА».

(TTA : district Staraja Sal'ja, raïon de Kijasovo, RO, 2014)

Kulikov A. N. : «Eh bien, j'avais un très bon ami. Il s'est donné la mort. Elle [la femme de l'informateur] aussi, il l'a appelée avec lui. Et voilà qu'il la tourmentait complètement, qu'il l'appelait, «On y va ». Après, j'ai accompli le rituel [littéralement : le lancement/le don en arrière] ; on ne l'a plus vu en rêve. » Kulikova Z.F. : «Il faut jurer, on dit, quand on accomplit le rituel de "don en arrière" : "Si tu es mort, couche-toi !" Tu accomplis le rituel de "don en arrière" en disant des gros mots, en jurant. "Je te prendrais bien avec moi, Arkadij [son mari], mais tu as encore du temps", disait [le mort]. [...] J'ai tout simplement jeté derrière mon dos, au cimetière, comme ça, en disant: “Trouve-toi une amie là-bas ! J'ai encore beaucoup de choses sur cette terre !" "

L'utilisation dans ce contexte précis d'un lexique expressif, qui prend la forme de jurons, est expliquée par B.A. Uspenskij par le fait que «les jurons expulsent la force impure du monde humain, visible, dans son espace "impur", c'est-à-dire "chez elle" " (cité par Steingold, 2006, p. 49-50). De surcroît, Aleksandra Steingold remarque :

En outre, des documents attestent que dans le milieu paysan de la Russie d'avant la révolution, on attribuait aux jurons une plus grande efficacité qu'à la prière protectrice chrétienne.

(Steingold, 2006, p. 137)

Il convient de remarquer que l'utilisation du lexique vulgaire russe (comme moyen apotropaïque) est un phénomène récent dans la culture oudmourte ${ }^{5}$. Les actions rituelles propitiatoires doivent être vues, d'une part, comme le moyen le plus archaïque d'établir le contact avec le monde de l'autre côté et, d'autre part, comme un outil apotropaïque. En sont témoins aussi bien les études de sources bibliophiliques (par exemple, Ostrovskij, 1873, p. 39 ; Smirnov, 1890, p. 189 ; Elabužskij, 1895, p. 800), que les recherches contemporaines (Šutova, 2001, p. 132-139; Petruhin, 2003, p. 250-251). Dans la culture traditionnelle des Oudmourtes, on entreprenait des manipulations de ce genre pour échapper à diverses maladies :

Amadouer les responsables de la maladie est l'un des moyens les plus archaïques de la lutte prophylactique dans la médecine populaire des Oudmourtes. [...] Dans la période contemporaine, cette tactique de communication ou d'adresse à l'esprit de la maladie a presque entièrement été supplantée par des méthodes qui en sont l'antithèse absolue : l'expulsion ou la destruction, clairement exprimées dans la tradition vivante des incantations verbales des Oudmourtes.

(Panina, 2014, p. 55)

Les paramètres spatiaux et les directions comme сьӧРЛАНЬ/БЕРЛАНЬ, «vers la limite, vers l'arrière » et ПАЛЛЯН/ПАЛЛЯН ПЕЛЬПУМ СьӦР « le côté gauche/la partie postérieure de l'épaule gauche " dans les exemples cités plus haut sont associés au monde des morts et c'est précisément pour cette raison qu'ils sont utilisés lorsque la communication avec le monde de l'autre côté devient nécessaire.

Dans la culture des Oudmourtes, il existe un autre présage curieux de l'approche de la mort: l'apparition inexpliquée de bleus sur le corps d'un individu. D'après les remarques des informateurs, ces signes d'une mort prochaine apparaissent principalement chez les personnes âgées, déjà proches du passage vers l'autre monde. Dans la culture traditionnelle des Oudmourtes, ce phénomène a reçu les noms suivants : 
ШӧЙ/ШэЙ чЕПЫЛЬТЭМ/КУРччЕМ, « pincement/piqûre de cadavre »

кУлэм МУРТ чЕпЫЛЬТЭМ, « pincement de défunt »

кУлэм КУРччЕм, « piqûre de défunt »

УБЫР/УБИР чЕпЫЛЬтЭМ, « pincement de vampire »

ВЕдйНь чЕПЫЛЛЯМ, « pincement de sorcières »

ШАЙТАН чЕПЫЛЛЯМ, « pincements de Šaitan »,

et également, d'après l'étude de G.E. Vereščagina, кУлэм мурт Родня чЕпыльтӥз,

c'est-à-dire qu'un parent défunt a pincé.

(Vereščagina, 1995, p. 80)

D'après les renseignements d'une informatrice du district Starye Kaksi, raïon de Možga, RO, de telles taches apparaissent surtout la nuit, lorsque la personne s'est couchée sans avoir prié au préalable (TTA : district Starye Kaksi, raïon de Možga, RO, 2014). Une autre informatrice a expliqué que cela se produit, si la personne «Ö॰Тэтэк изьыны выдэ», « si elle se couche [littéralement] sans Dieu », c'est-à-dire sans avoir prié Dieu (TTA : Iževsk, $\mathrm{RO}, 2014])$.

De nos jours, les Oudmourtes accordent une grande importance aux signes qui touchent au pied ou au talon d'un individu :

ПАлЛЯн ПыдПЫдэс лыдэ кЕ, кУлэм иворлы

(TTA : district Dubrovskij, raion de Kiasovo, RO, 2014).

Si le pied gauche démange, alors cela annonce une mort.

ПАлЛян Пыдтыш лыдэ кЕ, кУлэмЛЫ лУэ

(TTA : district Dubrovskij, raïon de Kiasovo, RO, 2010)

Si le pied gauche démange, cela annonce un décès.

Le pied, le talon, la partie inférieure de la jambe, c'est-à-dire les limites inférieures du corps humain, à travers lesquelles se réalise le contact avec le monde extérieur (« l'autre » monde), sont liés aux représentations de la mort (Mazalova, 2001, p. 42-46). En outre, selon la remarque de S.M. Tolstaja :

Les caractéristiques spatiales, où la gauche s'oppose à la droite, et est le signe de la sphère de l'autre côté, sont considérées comme appartenant au démoniaque, associées au malheur et au meurtre, et se trouvent en opposition avec la vie.

(Cité par Popova, 2011, p. 158)

En règle générale, les phénomènes cités plus haut sont interprétés par les Oudmourtes comme des avertissements et annoncent, pour un avenir proche, soit des changements essentiels dans la vie, soit la mort de quelqu'un.

Parfois, lorsqu'ils désirent savoir si une personne est attendue par la mort dans un futur proche, les Oudmourtes font de la divination. Ainsi, les Oudmourtes du raïon de Krasnogorsk de la République d'Oudmourtie accomplissent une divination particulière lors de la semaine sainte. Ils nouent à une souche un morceau de fil ou un bout de tissu qu'ils ont attaché à leur poignet la veille du jeudi saint, et ils jettent la souche dans la rivière. Ils observent ensuite ce qui se passe; si la souche coule, on doit s'attendre à la mort, si elle se fait emporter par le courant, on va vivre (NII et GGPI, 1987). 


\section{Sur le lit de mort}

Quand ils sont témoins de l'agonie d'un mourant, ses proches allègent ses souffrances, juste avant la mort, en accomplissant une suite de procédures qui permettent à l'âme de se libérer rapidement du corps :

- on dépose le mourant sur le sol, après avoir, au préalable, étalé de la paille, ou une matière inutile, une flanelle ou bien les bannye veniki, les rameaux que l'on utilise dans l'étuve. On considérait que la personne ne pouvait pas laisser partir son âme sur un matelas ;

- on ouvre le clapet du poêle ou on entrouvre la fenêtre, la porte ;

- on défait tous les nœuds des vêtements du mourant, on les déboutonne ;

- on place, sur l'appui d'une fenêtre ou sur une chaise à côté du mourant, une tasse avec de l'eau, en supposant que l'âme s'y baignera après sa sortie du corps ;

- on suspend une serviette propre, pour que l'âme s'essuie après son bain ;

- dans certaines traditions locales, lors de souffrances difficiles, on place un couteau à côté du mourant pour que l'âme soit rapidement libérée ;

- on allume une chandelle.

Toutes ces actions portent une profonde charge sémantique et symbolique.

Le sol est lié « à la représentation de la terre comme utérus/matrice, où se sont reposés les morts, où ils sont nés à nouveau, pour revenir à la vie, grâce à la sainteté de la Terre-Mère » (Éliade, 1999, p. 248-249).

(Pour comparaison : en Inde, dans la cérémonie funéraire, " on dépose tout de suite après la mort le cadavre sur le sol, "pour que l'âme puisse trouver plus facilement le lieu de séjour des morts", qui est situé sous la terre » [Gennep, 1999, p. 138]).

Le système d'actions utilisées pour l'apaisement du décès d'un individu comprend encore des gestes tels que le dévoilement, l'ouverture, le dénouement, le déboutonnage, et autres. Nous pouvons remarquer que des actions analogues sont utilisées pendant les accouchements difficiles. Ces analogies s'expliquent par les principes de la magie homéopathique : le fait d'ouvrir, de dénouer, de déboutonner, permet un écoulement libre de l'énergie, sa libération. Ainsi, le poêle, étant le centre de l'espace d'habitation, sert de point d'appui, «à travers lequel la mort arrive et l'âme s'en va dans l'autre monde » (Galdanova, 1980, p. 95-96).

L'eau utilisée dans ce contexte symbolise le voyage dans le monde des ancêtres. Elle sert aussi de médiateur, permettant l'ouverture ou la fermeture de la frontière symbolique. En outre, l'eau est une substance sacrée, qui a une fonction purificatrice. Par correspondance, c'est de là que vient l'habitude de couvrir ou de vider toute l'eau qui se trouve dans la maison, lorsqu'il y a un défunt. L'utilisation d'une serviette montre la signification symbolique du chemin vers la foule des ancêtres dans le lointain, qui attend l'âme. On utilise un couteau en métal comme protecteur de l'influence des êtres mauvais (à comparer avec le fait que, dans la culture de nombreux peuples, on dépose à côté du nouveau-né un couteau/des petits poignards, en tant que protecteurs). Dans notre cas, la propriété tranchante du couteau remplit la fonction d'objet symbolique qui sépare l'âme du corps. L'élément feu, représenté ici par la flamme de la chandelle, reçoit une signification apotropaïque. A.K. Bajburin relie l'allumage de la chandelle «aux représentations des ténèbres du royaume des morts, vers lequel se rend le mourant » (Bajburin, 1993, p. 104-105). 
Après avoir accompli ces actions, on laisse le mourant seul pendant un moment puis on instaure un silence complet. Il est interdit de pleurer ou de se lamenter. On considère qu'une expression bruyante de chagrin incommode l'exhalaison de l'âme et la rend difficile. À travers cette interdiction, on observe également une communion du mourant avec le monde des morts ; ainsi le silence est l'indice du monde de l'autre côté, silencieux. La tradition de laisser seul le mourant se rencontre également chez d'autres peuples finno-ougriens. Par exemple, chez les Maris, on laisse seul avec lui-même (avec la mort), sur son lit de mort, celui qui est en train d'exhaler son âme, dans la mesure où on croit que son regard est dangereux. On considère que la personne qu'il regardera juste avant de mourir mourra à sa suite ${ }^{6}$.

Si la mort atteint un individu de façon soudaine et sans grandes souffrances, on parle populairement de «КАПЧИ/КАНЬЫЛ эЗЕЛЬ», « une mort facile ». Règne l'idée que sont dignes de la mort facile les gens ayant eu une conduite exemplaire dans leur vie.

La mort des personnages marginaux suscite des représentations allant dans le sens inverse. On considère que ceux qui subissent, plus que d'autres, des souffrances physiques lors de l'exhalaison de l'âme sont des personnes ayant commis de mauvaises actions (sorciers ou sorcières, meurtriers, etc.), «lors de la mort desquels même le temps se gâte (УРод АДямИ КУЛЫКУ, КУАЗЬ НО УРОДъЯСьКЕ) » (Hristoljubova, 1984, p. 85).

Dans ce cas, on dit :

ЭзЕлЕз УГ вУЫ.

«Sa mort n'arrive pas.»

лӥл БАСьтйсь УГ лыкты

(TTA : Urazgil'dy, raïon de Tatyšly, RB, 2015)

«Le preneur d'âme n'arrive pas.»

лулЫЗ ПОтЫН ТУРТтЫСА КУРӒ̈ӞЕ

(TTA : district Staraïa Sal'ja, raïon de Kijasovo, 2014)

"Son âme souffre, elle essaye (littéralement : de sortir [du corps]).»

On croit que l'âme de celui qui a possédé des capacités magiques (qui est entré en communion avec le monde des morts) ne peut pas quitter son corps avant que la force surnaturelle soit transmise aux descendants (ou à n'importe quel autre individu). Pour libérer l'âme d'un sorcier ou d'une sorcière, les Oudmourtes connaissent un certain nombre de moyens gestuels. J'ai pu en noter quelques-uns chez les Oudmourtes du Sud :

\footnotetext{
МЫНАМ КАРТЭЛЭН АНАЕЗ ВЕДӤНЬ ВАУ. СОУЭН СУЗЭРЕЗ КУРАДӞИЗ ШУЭ НО. ТАТСЫ ИК, СЮЛМАЗ, МАРЫМЕН БЫШКАЛТӤЗЫ ШУИЗ, ВЕНЕН. НУ КУЛЫНЫ КУРАДӞИСЬ МАРЕКЕ, НОКЫЗЬЫ НО КУЛЭМЕЗ УГ ЛУ, ПЕ, НО. И ТАЗЫЫ «ПЫРС!», ПЕ, БЫШКАЛТӤЗЫ. СЭРЕ КУЛЭМ. А КУДӤЗЛЭН ВАЛ СИЕС ПОНО, ЛЭСЯ, ЧЫРТӤЯЗ. СИЕС ПОНО, ПЕ, ЧЫРТӤЯЗ, МЕД КУЛОЗ ШУСА.

(TTA : Starye Kaksi, raïon de Možga, RO, 2014)

«La mère de mon mari était sorcière. Sa sœur [quand la mère sorcière était mourante] a souffert, elle dit. D'abord ici, dans le cœur, on l'a piquée avec cette même aiguille, elle dit. Eh bien, celle qui souffre de mourir, [elle] ne peut mourir d'aucune façon. Et puis, "tyrs !", on l'a piquée. Puis elle est morte. Et certains, on leur accroche à la joue un collier d'épaule, il paraît. On accroche un collier d'épaule, oui, sur la joue, pour qu'elle meure. »
}

Cet exemple montre que, pour les personnages marginaux, on utilise des objets non standard (l'aiguille et le collier d'épaule) pour faciliter la libération de l'âme. L'aiguille, comme les autres objets en métal, est un objet apotropaïque qui protège des esprits impurs et possède également un effet neutralisant et apaisant. (Pour comparaison : dans la culture des Oudmourtes, pour se convaincre s'il se trouve dans l'isba un sorcier 
ou une sorcière, on enfonce dans le seuil de la porte une aiguille sans chas.) Un autre attribut magique est le collier d'épaule: "On utilise le collier d'épaule avec différents objectifs, mais dans tous les cas il est un symbole de la frontière, de la limite » (Goleva, 2011, p. 136). De là vient, assurément, l'utilisation du collier d'épaule pour le transfert de l'âme d'un état à un autre, d'un monde à l'autre. Par exemple, les Komis permiaks l'ont utilisé dans diverses pratiques magiques: pour neutraliser l'influence d'êtres mythologiques, pour guérir, pour recevoir des connaissances magiques, et pour dominer des personnages démoniaques (Goleva, 2011, p. 135-136).

Un fait curieux a été noté par le savant finlandais Uno Holmberg au début du xxe siècle, pendant ses expéditions de terrain chez les Oudmourtes en 1911: "Ce n'est que des grands sorciers qu'ils [les Oudmourtes] disent que, lorsqu'ils meurent, s'élève un tourbillon ou une tempête » (Putešestvija, 2014, p. 106).

Lorsqu'ils font face à un tourbillon, les Oudmourtes prononcent la plupart du temps une formule verbale universelle,

ТӤРЕ-ПУРТэ КИЯМ !, « mon couteau-hache dans mes mains ! »

ou

ТӤРЕ-ПУРТЭ БОРДАМ, « mon couteau avec moi ! »

(Gataullina, 2008, p. 128)

Cette formule, selon la conception populaire, doit protéger de la force mauvaise qui s'échappe du corps du sorcier ou de la sorcière sous l'aspect d'un être non personnifié.

Selon les informations collectées auprès d'informateurs oudmourtes (raïon de Tatyšly, $\mathrm{RB}$, raïon de Kiasovo et de Možga, RO) et maris ${ }^{7}$, pour faciliter la séparation de l'âme d'une sorcière, il est indispensable d'enlever/d'ôter le konëk ${ }^{8}$ du toit de la maison. Ce fait est noté également par D.K. Zelenin, chez les Slaves de l'Est. Le savant suggère que : «Probablement, cette habitude prend ses racines dans les temps où les maisons avaient des toits, mais n'avaient pas de plafonds » (Zelenin, 1991, p. 346).

D'après les récits d'une informatrice du raïon de Malopurga de la RU, une sorcière $a$, sous la peau au niveau du plexus solaire, quelque chose qui a l'aspect d'une toile (ДэРА КАДЬ). Elle ne peut mourir que lorsque ce tissu même est réduit en poudre (ТTA: Malaja Purga, UR, 2014). Il semble donc que le terme de la vie de tels personnages est déterminé, nominal, et dépend d'une matière magique, reçue (possiblement, de la sphère de l'autre monde), pendant l'apprentissage de l'art de la magie.

\section{La « rencontre » avec la mort}

D'après les matériaux de terrain de l'auteur, l'individu se transforme physiquement et psychologiquement avant la mort: ses yeux se voilent, ses mains deviennent froides, son visage pâlit, parfois il cesse de reconnaître ses proches, de distinguer le jour de la nuit, il devient agressif, nerveux, etc.

Un individu proche de la mort acquiert les propriétés et les qualités d'un personnage liminal, c'est-à-dire qu'il se trouve à la limite entre le monde des vivants et le monde des morts. Il peut voir les êtres de l'autre monde et entrer en rapport avec eux, et son âme est capable de s'envoler du corps et de voyager. On considère qu'il arrive que les membres de la famille puissent voir l'âme d'un individu qui est gravement malade ou mourant. Dans une telle situation, les Oudmourtes disent :

ИШАНЗЭ ВОЗЬМАТӥз/АДӞЫтӥз.

«Il a montré son fantôme/son ombre. » 
Dans la conscience traditionnelle, "l'apparition de personnages mythologiques se comprend comme un signe du rapprochement de l'individu avec le monde des ancêtres » (Goleva, 2011, p. 119).

C'est pourquoi les Oudmourtes supposent que si un individu malade voit des morts, ou s'ils apparaissent devant lui, et qu'il entre en dialogue avec eux, c'est qu'il n'a plus longtemps à vivre. Pratiquement tous les informateurs rapportent que les membres de la famille qui sont décédés "viennent " lui rendre visite, pour rencontrer l'âme du défunt et la conduire ou l'emporter dans l'autre monde. On peut trouver de semblables représentations chez de nombreux peuples. Ainsi, on considère chez les Tchouvaches que «les parents de l'autre monde, la veille de la mort d'une personne qui leur est proche, viennent la nuit, depuis le cimetière jusqu'au village, sous l'aspect d'un train nuptial» (Salmin, 2007, p. 235). Chez les Besermans, « les parents et les membres de la famille qui sont morts "rencontrent" et "proposent leur aide" à leur parent au moment du transfert vers le monde sous terre " (Popova, 2011, p. 158-159).

C'est précisément ainsi que s'expliquent les hallucinations visuelles et la conversation avec les parents décédés en face de la mort :

МЕМЕЙ УЙСЫРМИЗ КЕ, ЧИСТО ВЕРАСЬКЕ ВАЛ. ПЕТЕН НО ВЕРАСЬКЕ ВАЛ И ДЯДЕН НО ВЕРАСЬКЕ. МАРЫМ, ШУЭ: «Я, МЫНО ИНЬ, МЫНО. МОН ДАСЯСЬКЕМЫН ИНЬ, СКОРО ВУО. ТӤ ДОРЫ МЫНО ИНЬ». ОЗЬЫ ИНЬ СО ВЕРАСЬКЕ. «КЫЧЧЫ-О МЫНОД, МЕМЕЙ?»ШУКО НО. «МА, ПЕТЫР ӦТИЗ НО, ОЧЧЫ МЫНО», - ШУЭ. СОБЕРЕ НОШ ИК ШУЭ, ӜАМДЭ УЫСА: «ОЙДО ИНИ ЧАЛЯК!» «МА, АЛИ ИК, ДАСЬКИ КЕ, МЫНО ИНЬ», - ШУЭ СО НОШ. СО АӞӞЕ ИНЬ, КУЛЭМ МУРТЪЁСЫЗ АӞӞЕ ИНЬ. ӦТЁ ИНЬ СЭ».

(TTA : Gurez'-Pudga, Vavož raïon de Vavož, RO, 2013)

"Quand maman a commencé à délirer [à cause de sa maladie douloureuse], elle conversait [avec tous les morts]. Et elle discutait avec Petia [le défunt frère de la mourante], et avec papa. Elle dit ça : "D'accord, je viens tout de suite, je viens. Je suis déjà prête, j'arrive bientôt. J'arrive auprès de vous." Voilà ce qu'elle raconte. "Où est-ce que tu vas, maman ?" je lui demande. "Eh bien, Petia m'a appelée, je vais là-bas", elle dit. Puis elle dit à nouveau, un peu plus tard: "Allez, plus vite !" "Eh bien, maintenant, je me prépare, j'arrive”, dit-elle à nouveau. Elle voit déjà, elle voit déjà les morts. Ils l'appellent déjà. »

D'après les récits des informateurs, les parents morts «qui sont arrivés » ne peuvent être vus que par un individu gravement malade ou sur son lit de mort.

Dans la tradition des Oudmourtes, on rencontre assez fréquemment d'autres croyances prédisant une mort prochaine. Nous avons pu noter, chez nos informateurs d'outre-Kama, des idées semblables, selon lesquelles il est parfois possible d'observer visuellement les âmes mortes, par exemple sous la forme de petits oiseaux :

БАКЧАЙЫН ГУ ВАНЬ. СО ГУ ДОРЫН ПУКСЁНТӤЕЗ ВАНЬ ГАРИФАЛЭН, ОЧЧЫ МЫНЭМ. ВӦЗАЗ ЛЕМПӰ ВАЙ СЭЗЪЯСЬКЫСА КЫЛЛЕ ТАЗЬЫ. ӞОЛЬГЫРИ ТУСЪЕМ ПУЖИЕЗ, ТЫЛОБУРДОЕЗ БАӞӞЫНГЕС, ӞОЛЬГЫРИ КАДЬ НО ВЫЛЙЙЫЗ. [...] СО ТЫЛОБУРДОЁС МЫНЫСА ПУКСИЛЛЯМ НО ОЧЧЫ, ВӦЗАЗ ИК КЫЛЛЁ. «БОН, ТАОСЫЗ НЕНЬ СЁТЪЯСА ДЫШЕТӤДЫ-А? МАР ТА ТАШЕ?» - ШУСЬКО. «ОЛО, КУЛЭ КЕ, ЛӰЛ БАСЬТӤСЬЁС ЛЫКТӤЛЛЯМ, ОЛО, АНАЙЁСЫД ЛЫКТӤЛЛЯМ», - ШКО. ЧИРДО, ТЭЧЧАЛО, СО ЛЕМПӰЫН КЫЛЛЁ. УГ НО КЫШКАЛО. [...] КАЗЫР БЕРТЫКЫ, СО ТЫЛОБУРДОЁС СЕРЕ ЛЫКТӤЗЫ. [...] «МАР ТЫЛОБУРДО МЕДА ТА? ОЛО, АНАЙЁС ЛЫКТӤЛЛЯМ-А МОНЭ НУЫНЫ?» - ШУЭ ГАРИФА. «ЧИРК! ЧИРК! ЧИРК!» ШУСА ТЭЧЧАЛО КОТЫРАЗ.

(TTA : Novye Tatyšly, raïon de Tatyšly, RB)

«Dans le potager il y a une fosse. À côté de cette fosse il y a le banc de Garifa, [elle] est allée là-bas. À côté, se balance la branche d'un merisier à grappes. Il a la même couleur que les moineaux, [mais] cet oiseau est plus grand, son dos est comme celui 
du moineau. [...] Ces oiseaux ont volé directement jusque-là et se sont posés à côté. “Alors, c'est au pain qu'ils se sont habitués, [ces oiseaux] ? Qu'est-ce que c'est que ça ?" je dis. "Ou alors, si je dois mourir, les [esprits] Preneurs d'âme sont venus, ou mes parents [défunts] 'sont arrivés"', je dis. Ils gazouillent, ils bondissent, ils se posent sur ce merisier. Et ils n'ont pas peur. [...] Quand nous sommes rentrés à la maison, ces oiseaux nous ont suivis. [...] "Qu'est-ce que c'est que ces oiseaux ? Ou est-ce que mes parents [défunts] sont venus pour moi ?" demande Garifa. "Tchip! Tchip ! Tchip !" ils gazouillent, bondissent autour d'elle. »

Représenter l'âme par une figure ailée est caractéristique de nombreux peuples du monde, ce qui revêt un profond sens cosmologique, mythologique et cultuel, et reflète l'univers mental du peuple. Selon les conceptions religieuses et mythologiques des peuples finno-ougriens, les forces divines et les ancêtres défunts sont personnifiés sous des traits ornithomorphes; il s'agit de l'une des variantes de leurs apparitions physiques dans le monde des humains. En outre, selon les représentations populaires, les personnages marginaux, qui possèdent des connaissances magiques, peuvent se transformer en oiseaux. Ainsi, chez les Slaves :

Les âmes des gens justes ou des ancêtres vénérés sont symbolisées par la figure d'oiseaux «purs» (la colombe, l'hirondelle, la cigogne, l'alouette, et d'autres), tandis que les oiseaux « impurs» (le hibou, le grand duc, le corbeau) endossent le rôle de défunts, de pécheurs, de sorciers.

(Cité par Usačeva, 2008, p. 173)

De là, sans doute, vient la représentation des Oudmourtes selon laquelle, si une pie vient à la maison, il y aura des invités ou une autre nouvelle ; si c'est un corbeau, c'est un mauvais présage, un décès; si c'est un coucou, c'est un incendie ou un malheur, tandis qu'une colombe blanche qui s'envole dans le cadre de la porte symbolise le bonheur et la grâce divine. Dans la tradition oudmourte, la figure ornithomorphe se rencontre également dans la poésie nuptiale ou celle du départ à l'armée, où elle s'associe respectivement avec la fiancée ou le fiancé, et avec le conscrit (voir Vladykina, 2006, p. 50-57).

Dans le groupe des Oudmourtes d'outre-Kama, qui ont été en contact étroit avec des peuples turcs et ont été exposés à l'influence musulmane, il existe un personnage particulier, l'ange de la mort, Azrali ${ }^{9}$. On rencontre également un autre nom, «лыл/ӟAH БАСьтӥсь», « le Preneur d'âme » (Sadikov, 2008, p. 154). Les Maris de la même région ont également des représentations d'un être de l'autre monde, Azyren ${ }^{10}$. Chez les Tchouvaches, l'esprit qui emporte l'âme s'appelle Čun illi ${ }^{11}$, (littéralement : «l'Enleveur d'âme ", traduction de Salmin) ou Esrel ${ }^{12}$ (de l'Azraïl musulman, commentaire de Salmin ; Salmin, 2007, p. 238). Dans les représentations populaires, l'ange de la mort a une figure anthropomorphe. Selon les Oudmourtes, il arrive auprès d'un individu mourant sur un cheval gris et, selon les Maris, sur des chevaux noirs dans un tarantass ${ }^{13}$. Ses attributs essentiels sont des couteaux tranchants ${ }^{14}$, à l'aide desquels il sépare l'âme de l'individu de son corps physique. Comme le remarquent les témoins maris, l'ange de la mort peut être vu par « les gens pécheurs ou méchants; il se présente rarement aux gens justes ", parfois il apparaît à un individu pendant l'exhalation de l'âme, ou on peut le rencontrer en rêve (Toidybekova, 1997, p. 302 ; Jamurzina, 2011, p. 136). Selon la vision d'une informatrice du village de Novye Tatyšly, raïon de Tatyšly, RB, l'agent de la mort, лӥл БАСьтйсь, peut avoir une figure ornithomorphe, prendre la forme d'un petit oiseau, qui ressemble au moineau, et il se montre à l'individu devant la mort (TTA, village de Novye Tatyšly, raïon de Tatyšly, RB, 2014). Un personnage ornithomorphe analogue a été noté en Oudmourtie du Nord, à Ujvaj, raïon de Debëssy, RO, («лул 
БАСБтӤсь ТЫЛОБУРДО», " un oiseau qui prend l'âme »), qui est d'une couleur jaune et vient auprès du mourant avant sa mort (Kel'makov, 2006, p. 191).

D'après les récits de nos informateurs, il y a une différence entre les défunts morts d'une mort naturelle (atteints d'une maladie grave, des personnes âgées), et ceux qui sont morts d'une mort non naturelle (des conséquences d'un accident, tués ou autres). $\mathrm{Au}$ second groupe appartiennent aussi les malades mentaux. D'après la définition de D.K. Zelenin, ceux qui sont morts de leur belle mort atteignent le rang des ancêtres, tandis que « les gens qui sont morts avant le terme de leur mort naturelle, qui meurent souvent dans leur jeune âge, d'une mort brutale, malheureuse ou violente » (Zelenin, 1995, p. 39) comptent parmi les morts impurs. Dans le premier cas, le mourant, sentant l'approche de la mort, appelle ses parents et les bénit, leur dit des mots d'adieu. Dans de rares cas, il exécute une chanson qu'il a lui-même composée, pour qu'après sa mort ses parents la chantent et se souviennent de lui. Dans le cas où c'est un membre âgé de la famille qui meurt, on s'adresse à lui avec cette prière :

шуддэ сёт

(TTA : district Dubrovskij, raion de Kijasovo, RO, 2008)

" Donne ton bonheur. »

On pense qu'un individu qui a reçu le bonheur d'un défunt reçoit en même temps son patronage et son aide dans les situations difficiles de la vie ${ }^{15}$. Les âmes des gens morts de mort rapide, sans en avoir conscience, sont considérées comme dangereuses. Elles ne se rendent pas dans le monde des morts, mais « elles errent à travers la terre, [...] effraient et terrifient les proches, leur causent des dommages, envoient des maladies, servent les démons, se mêlent à une force impure »(Zelenin, 1995, p. 23).

Figure 1

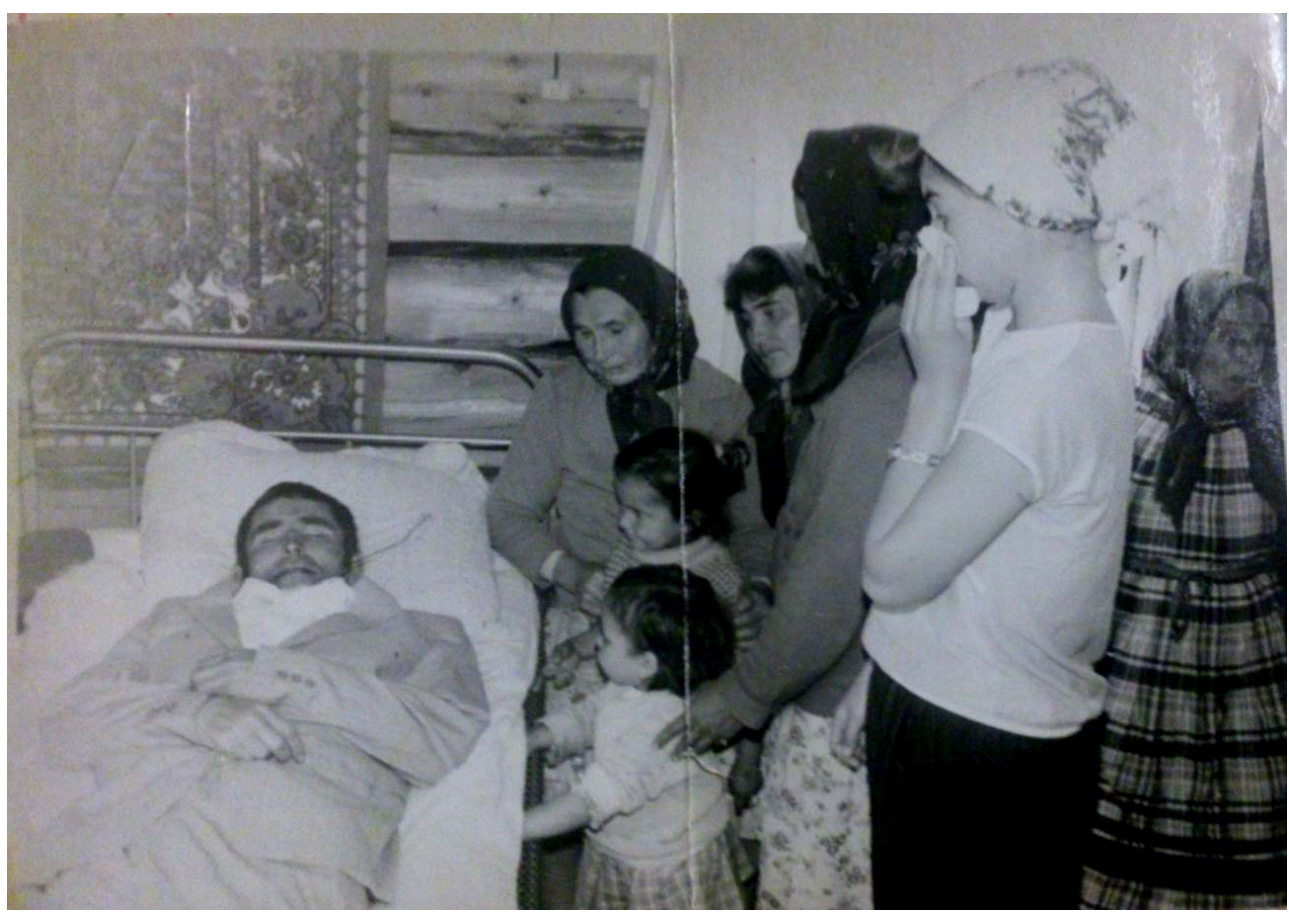

Les adieux de la famille au défunt sur son lit de mort (village de Vjazovka, raïon de Tatyšly, RB, 1992) (c) Archives familiales de la famille Timerhanov 


\section{La « visite » dans l'autre monde}

Dans les sources publiées et dans mes matériaux de terrain, on rencontre l'idée d'une "visite " temporaire de l'autre monde. On considère que cela arrive aux gens qui se trouvent dans une syncope profonde, dans le coma, ou qui sont dans un sommeil léthargique :

КУЛЭм КАДЬ КЫЛЛЬЫны, « séjourner comme un défunt ", кулыса кылльыны, « séjourner après être mort ».

Les Oudmourtes d'outre-Kama

disent de telles personnes qu'elles sont « allées dans le nert » (du tatare MэPT, mert, «le monde souterrain, d'outre-tombe »), нэртэ мынэм. De telles personnes gardent sur le corps, le visage ou les mains des marques particulières qui ont l'aspect de brûlures.

(Sadikov, 2008, p. 156)

Dans la tradition oudmourte, on appelle les phénomènes de ce genre кулысА улӟон/ кулэмысь улӟыса султон, «la renaissance après la mort». Conformément aux perceptions des informateurs, il n'est pas rare que ceux qui sont allés dans le monde souterrain reçoivent des capacités magiques et deviennent des magiciens. Comme le remarque S.M. Tolstaia, «la relation à ces gens, que ce soit pendant leur vie ou après leur mort, demande toujours d'être sur ses gardes » (Tolstaia, 2010, p. 235).

Un chercheur écrit que, dans la tradition slave, on accordait dans la plupart des cas une connotation négative à cette fausse mort, liée à la sémantique péjorative de la répétition :

Pendant leur vie ils sont vus comme dangereux, dans la mesure où ils sont venus deux fois dans « ce » monde, qu'ils ont franchi deux fois la frontière entre la vie et la mort (à leur naissance et à leur fausse mort), et après la mort ils sont encore dangereux, parce qu'ils sont morts deux fois.

(Sadikov, 2008, p. 156)

En lien avec cette allégation, un des faits notés dans le district Bol'šie Siby, raïon de Možga, RO, d'après les souvenirs des anciens habitants :

ПИЛЬМОН ПЕДЯГАЙЛЭН АНЭЗ КУЫСА... [...] ПЕДЯГАЙЛЭН АНЭЗ ИНЬ СО. ПЕДЯГАЙЛЭН ТАРЕ КУ КУЭМЕЗ ИНЬ, МИ ПИЧИЕСЬ ДЫРЪЯ СО ПЕРЕСЬ ВАУ ТУЖ. [...] ПИЛЬМОН ПЕДЯГАЙЛЭН АНЭЗ КУЛЭМ НО, НУНАЛАЗ ВАТНЫ... МАЛЫ ВАТӤЛЛЯМТЭ, ЧЕРКЫН кӧЛтӤЛЛЯМ. УЫӞЫЛЭМ, ПЕ, ШУСА ВЕРАСЬКЫЛӤЗЫ. ДЙСЬКУТЭЗ, МАРЫМ, ОШИСЬКЕМ, ПЕ, ВЫЛЭМ ШАЙПУЛ ВӦЗТӤ. ВИИЛЛЯМ-А МАР-А? БАӴКА ВИЕ-А СЭ, КЫЗЬЫ? ТӤНЬ ОЗЬЫ ВЕРАСЬКОН ВАУ. КУЛЭМ МУРТЭ, ПЕ, УЛӞЕ КЕ, УЛӞЫН, ПЕ, УГ ЛЭЗЁ НИ, БЕРЕН, ПЕ, ВИЁ.

(TTA : district Bol'šie Siby, raïon de Možga, RO, 2014)

« La mère de tonton Fedia Pil'mon ${ }^{16}$ est morte... [...]. Elle est déjà la mère de tonton Fedia. Combien de temps s'est passé depuis la mort de tonton Fedia, quand on était jeunes, il était [alors] déjà très vieux. [...] La mère de tonton Fedia Pil'mon est morte, oui, ce jour-là on devait l'enterrer... Pourquoi on l'a pas enterrée, on [l']a déposée dans l'église la nuit. Elle était vivante, elle parlait. Ses vêtements pendaient, oui, le long de la tombe. On l'a tuée, quoi ? Est-ce que le prêtre la tue, quoi ? Voilà ce qu'on racontait. Quelqu'un qui est mort, s'il revit, s'il vit, eh ben, on ne le laisse pas, on le tue une seconde fois. "

La coutume décrite ci-dessus, de tuer un "mort " revenu à la vie, a été accomplie par un prêtre, c'est-à-dire par un étranger venant d'une autre culture (orthodoxe), où le phénomène se comprenait comme une anomalie, dangereuse pour les vivants. Les Oudmourtes, malgré leur appartenance extérieure à l'Église orthodoxe, ont gardé 
jusqu'à aujourd'hui encore des conceptions traditionnelles, principalement autour du culte des ancêtres - sans parler de la situation au XIX ${ }^{e}$ siècle. Les mentions du meurtre d'un mort revenu à la vie sont pratiquement absentes de la tradition orale des Oudmourtes, bien que mes informateurs affirment que, dans le passé, ces situations n'étaient pas rares. Certains, pendant leur vie, avertissent même leurs proches de bien s'assurer qu'ils sont morts :

КЫӴЕ КЕ НО НЫЛ-А МАРА ЗУНДЭССЭ ЫМАЗ ПОНЫСА НЬЫЛЫНЫ ШЕДЬТЭМ НО, ГУНЬДЭМ, ПЕ, НО, СОЕ, ПЕ, ВАТӤЛЛЯМ НО... СЫРЕ МАЛЫ БОН СОЕ ПОТТАНО ЛУИЛЛЯМ МЕДАК, УГ ТОСЬКЫ. КЫМИНЬ, ПЕ, ЛУЭМ, ПЕ, СО. [...] СОИН ИК АЗЬЛО КУНЬ УЙ КӦЛТЫТЭК УГ ВАТО ВЫЛЭМ АДЯМИЕЗ. КУНЬ УЙ КӦЛЭМ БЕРАЗ... ЯКЕ УЛӞОЗ ШУСА. МОН НО, МОНЭ КУНЬ УЙТЭК ЭН ВАТЭ ШУСА, СЕРЕКЪЯСЬКО. МОН ТАНИ КАМПЕТ АЛИ НО ЧУПЧИСЬКО ВАЛ ИЖЖОГАЛЭСЬ. [...] ИЗЬЫН ВЫДЫКЫМ НО, ЫМАМ ПОНӤСЬКО НО, СЕРЕКЪЯСЬКО: «КУНЬМОЙТЭК ЭН ВАТЭ МОНЭ, МЫНАМ ЯКЕ КАМПЕТЭ ШУНАСА БЫРОЗ.»

(TTA : district Dubrovskij, raion de Kijasovo, RO, 2013)

«Une fille avait pris dans sa bouche un anneau et l'avait avalé soudain, et, ben, on l'a enterrée... Ensuite, pourquoi on n'a pas creusé, je ne sais pas. Elle est tombée la tête en avant, on aurait dit. [...] C'est pourquoi autrefois on enterrait le défunt seulement après la troisième nuit. Après qu'il a dormi trois nuits, [qu'on l'enterrait] ... En pensant qu'il peut se réveiller soudain. Et je rigole, ne m'enterrez pas, [avant que] n'arrive la troisième nuit. J'ai tout juste sucé un bonbon à cause de mes brûlures d'estomac. [...] Quand je vais me coucher, je le mettrai aussi dans la bouche et je dirai: "Ne m'enterrez pas avant trois fois vingt-quatre heures, soudain le bonbon va fondre [et je revivrai]. »

\section{Conclusion}

Ainsi, dans la culture traditionnelle des Oudmourtes, la période qui précède la mort et les expériences d'avant-mort d'un individu sont l'étape initiale du voyage vers l'autre existence. L'individu lui-même reçoit les qualités et les propriétés d'un personnage liminal, c'est-à-dire que « le mourant devient déjà un mort » (Baiburin, 1993, p. 105).

Au travers de la destruction de l'état biologique, l'individu, qui se trouve à la limite des mondes, communique avec ses ancêtres morts et avec l'agent de la mort. Dans certains cas, il est possible de visiter l'autre monde et d'en revenir. Cependant, « dans toutes ces actions, jusqu'à la fin, le mourant agit dans le rôle d'un sujet, accomplissant un rituel, mais avec l'arrivée du décès, il devient l'objet du rituel » (Baiburin, 1993, p. 104).

Cela détermine la relation que son entourage noue avec lui, de même que le comportement communicatif que celui-ci tient à son égard, tandis que le mourant luimême acquiert un nouveau statut social et rituel.

Dans la culture populaire, la période d'avant-mort, qui se trouve être un moment critique, liminaire, définit les particularités du comportement verbal et non verbal tant de l'individu que de tout l'environnement social (l'entourage familial, la communauté villageoise). La mort, moment crucial du mélange des mondes, exige une stratégie déterminée, une tactique comportementale qui se fonde sur des formes travaillées depuis des siècles et des moyens servant à réguler le comportement d'un individu dans ce moment difficile de forte émotion. Il convient de remarquer que, généralement, l'individu n'a pas conscience des mécanismes qu'il met en œuvre dans la communication : il reçoit des « clichés » culturels prêts à l'emploi, et n'improvise, ou ne crée, que rarement des ajouts individuels sous forme de modèles verbaux et gestuels. 
C'est pourquoi il est si important de faire des recherches sur ce thème, en particulier maintenant, alors que la génération ancienne recourt encore à ces procédés pour permettre le rapport avec le monde de l'autre côté, et que chez les plus jeunes ces procédés revêtent assez souvent un caractère mécanique, ne manifestant aucune conscience des actions accomplies, qui sont, le plus souvent, inconscientes et souvent non reproduites.

Pour conclure, ajoutons que le processus même de passage d'un individu vers l'autre existence, qui s'exprime à travers ses expériences d'avant-mort, est un élément essentiel de la transformation du système rituel d'enterrement et de commémoration, qui exige des recherches plus poussées dans la mesure où les processus globaux contemporains peuvent influencer (ou influencent déjà) la thématique étudiée.

\section{BIBLIOGRAPHIE}

\section{Références bibliographiques}

BAJBURIN, 1993 = БАЙБУРИН АЛЬБЕРТ КАШФУЛЛОВИЧ, РИТУАЛ В ТРАДИЦИОННОЙ КУЛЬТУРЕ: СТРУКТУРНО-СЕМАНТИЧЕСКИЙ АНАЛИЗ ВОСТОЧНОСЛАВЯНСКИХ ОБРЯДОВ (Le rituel dans la culture traditionnelle : analyse structurelle et sémantique des rituels des Slaves de l'Est), САНКТ-ПЕТЕРБУРГ: НАУКА, 199, 223 С.

ÉLABUŽSKIJ, 1895 = ЕЛАБУЖСКИЙ МИХАИЛ СТЕФАНОВИЧ, «ОБРЯДЫ НЕКРЕЩЕННЫХ ВОТЯКОВ ЕЛАБУжСКОГО УЕздА» (Les rituels des Votiaks non baptisés de l'uezd d'Elabuga), ВЕВ, № 9, cC. $800-805$.

ÉLIADE, 1999 = ЭЛИАДЕ МИРЧЕА, ОЧЕРКИ СРАВНИТЕЛЬНОГО РЕЛИГИОВЕДЕНИЯ (Traité de l'histoire des religions), МОСКВА: ЛАДОМИР, 488 С.

GALDANOVA, 1980 = ГАЛДАНОВА ГАЛИНА РИНЧИНОВНА, «КУЛЬТ ОГНЯ У МОНГОЛОЯЗЫЧНЫХ НАРОДОВ И ЕГО ОТРАЖЕНИЕ В ЛАМАИЗМЕ» (Le culte du feu chez les peuples mongols et son reflet sur le lamaïsme), СОВЕТСКАЯ ЭТНОГРАФИЯ, № 3, сс. 94-100.

GATAULLINA, 2008 = ГАТАУЛЛИНА АНГЕЛИНА ИВАНОВНА, «АСТРОНОМИЧЕСКИЕ ВОЗЗРЕНИЯ УДМУРТОВ» (Les conceptions astronomiques des Oudmourtes), ЕЖЕГОДНИК ФИННО-УГОРСКИХ ИССЛЕДОВАНИЙ '07, НАУЧ. РЕД. Н.И. ЛЕОНОВ; СОСТ. И РЕД. А.В. ИШМУРАТОВ, Р.В. КИРИЛЛОВА; ОТВ. РЕД. Д.И. ЧЕРАШНЯЯ, ИЖЕВСК: УДМУРТСКИЙ ГОСУДАРСТВЕННЫЙ УНИВЕРСИТЕТ; ERGO, cС. 49-57. GENNEP, 1999 = ГЕННЕП АРНОЛЬД ВАН, ОБРЯДЫ ПЕРЕХОДА. СИСТЕМАТИЧЕСКОЕ ИЗУЧЕНИЕ ОБРЯДОВ (LeS rites de passage. Étude systématique des rites), ПЕР. С ФРАНЦ., МОСКВА: ИЗДАТЕЛЬСКАЯ ФИРМА «ВОСТОЧНАЯ ЛИТЕРАТУРА» РАН, 198 C.

GOLEVA, 2011 = ГОЛЕВА ТАТЬЯНА ГЕННАДЬЕВНА, МИФОЛОГИЧЕСКИЕ ПЕРСОНАЖИ В СИСТЕМЕ МИРОВОЗЗРЕНИЯ КОМИ-ПЕРМЯКОВ (Les personnages mythologiqes dans le système ontologique des Komis permiaks), САНКТ-ПЕТЕРБУРГ: ИЗДАТЕЛЬСТВО «МАМАТОВ», 272 С. 
HRISTOLJUBOVA, 1984 = ХРИСТОЛюБОВА ЛЮДМИЛА СТЕПАНОВНА, СЕМЕЙНЫЕ ОБРЯДЫ УДМУРТОВ (ТРАДИЦИИ И ПРОЦЕССЫ ОБНОВЛЕНИЯ) (Les rituels familiaux des Oudmourtes. Traditions et processus de renouvellement), ижЕВск: УдмУРтия, $128 \mathrm{C.}$

JAMURZINA, 2011 = ЯМУРЗИНА ЛЮДМИЛА, ОБРЯДЫ СЕМЕЙНОГО ЦИКЛА МАРИ В КОНТЕКСТЕ ТЕОРИИ ОБРЯДОВ ПЕРЕХОДА (НА ПРИМЕРЕ ВОСТОЧНЫХ МАРИ) (Les rituels du cycle familial chez les Maris dans le contexte des théories des rites de passage) sur la base de l'exemple des Maris orientaux), Tartu: Tartu Ülikooli Kirjastus, 219 c.

KARABEL'NIKOV = КАРАБЕЛЬНИКОВ ДМИТРИЙ, «„ПОХОРОНИТЕ МЕНЯ СТОЯ”: ЗАБЫТЫЕ ТАЙНЫ МОРдОвских погостов» (Enterrez-moi debout : les mystères oubliés des pogost mordves), элЕктРОнныЙ РЕСуРс: http://nn.mk.ru/articles/2015/06/05/pokhoronite-menya-stoya.html [consulté le 19 janvier 2017].

КЕL'MАКОV, 2006 = КЕЛЬМАКОВ ВАЛЕЙ КЕЛЬМАКОВИЧ, КРАТКИЙ КУРС УДМУРТСКОЙ ДИАЛЕКТОЛОГИИ: УЧЕБНОЕ ПОСОБИЕ ДЛЯ ВЫСШИХ УЧЕБНЫХ ЗАВЕДЕНИЙ (Bref cours de dialectologie oudmourte : manuel universitaire), 2-Е ИЗД., СТЕРЕОТИПНОЕ, ИЖЕВСК: ИЗДАТЕЛЬСКИЙ ДОМ «УДМУРТСКИЙ УНИВЕРСИТЕТ», 386 С.

КUSTOVA, 2009 = КУСТОВА ЮЛИЯ ГЕОРГЕВНА, «СНОВИДЕНИЯ И ПРИМЕТЫ, ПРЕДВЕЩАЮЩИЕ СМЕРТЬ У ХАКАСОВ» (Les rêves et les prémonitions annonçant la mort chez les Khakasses), СИБИРСКИЙ СБОРНИК 1: ПОГРЕБАЛЬНЫЙ ОБРЯД НАРОДОВ СИБИРИ И СОПРЕДЕЛЬНЫХ ТЕРРИТОРИЙ, КНИГА І, ОТВ. РЕД. Л.Р. ПАВЛИНСКАЯ, САНКТ-ПЕТЕРБУРГ: МАЭ РАН, СС. 49-53.

MAZALOVA, 2001 = МАЗАЛОВА НАТАЛЬЯ ЕЕГЕНЬЕВНА, СОСТАВ ЧЕЛОВЕЧЕСКИЙ. ЧЕЛОВЕК В ТРАДИЦИОННЫХ СОМАТИЧЕСКИХ ПРЕДСТАВЛЕНИЯХ РУССКИХ (La composition de l'homme. L'homme dans les représentations somatiques traditionnelles des Russes), САНКТ-ПЕТЕРБУРГ: «ПЕТЕРБУРГСКОЕ ВОСТОКОВЕДЕНИЕ», 192 C.

Mifologija komi, 1999 = МИФОЛОГИЯ КОМИ (La mythologie des Komis), Н.Д. КОНАКОВ, А.Н. ВЛАСОВ, И.В. ИЛЬИНА..., НАУЧ. РЕД. В.В. НАПОЛЬСКИХ, МОСКВА: ИЗДАТЕЛЬСТВО ДИК, 480 С.

OSTROVSKIJ, 1873 = ОСТРОВСКИЙ Д.Н., ВОТЯКИ КАЗАНСКОЙ ГУБЕРНИИ (Les Votiaks du gouvernement de Kazan), ТРУДЫ ОБЩ-ВА ЕСТЕСТВОИСПЫТАТЕЛЕЙ ПРИ КАЗАН. УН-ТЕ, КАЗАНЬ, Т. 4, ВЫП. І, 48 С. PANINA, 2014 = ПАНИНА ТАТЬЯНА ИГОРЕВНА, СЛОВО И РИТУАЛ В НАРОДНОЙ МЕДИЦИНЕ УДМУРТОВ: МОНОГРАФИЯ (La parole et le rituel dans la médecine populaire des Oudmourtes : monographie), НАУЧ. РЕД. Т.Г. ВЛАДЫКИНА; ИЖЕВСК, УИИЯЛ УРО РАН, 240 С.

PETRUHIN, 1995 = ПЕТРУХИН ВЛАДИМИР ЯКОВЛЕВИЧ, «ПОГРЕБЕНИЕ» (L'enterrement), СЛАВЯНСКАЯ МИФОЛОГИЯ. ЭНЦИКЛОПЕДИЧЕСКИЙ СЛОВАРЬ, МОСКВА: ЭЛЛИС ЛАК, 416 С.

PETRUHIN, 2003 = ПЕТРУХИН ВЛАДИМИР ЯКОВЛЕВИЧ, МИФЫ ФИННО-УГРОВ (Les mythes des Finno-Ougriens), МОСКВА: ООО «ИЗДАТЕЛЬСТВО АСТРЕЛЬ», 464 С.

POPOVA, 2011 = ПОПОВА ЕЛЕНА.ВАСИЛЬЕВНА, КУЛЬТОВЫЕ ПАМЯТНИКИ И САКРАЛЬНЫЕ ОБЪЕКТЫ БЕСЕРМЯН: МОНОГРАФИЯ (Les monuments cultuels et espaces sacrés des Bessermans), НАУЧ. РЕД. Г.А. НИКИТИНА, ИЖЕВСК: УИИЯЛ УРО РАН, 320 С.

Putešestvija, 2014 = ПУТЕШЕСТВИЯ К УДМУРТАМ И МАРИЙЦАМ. ПИСЬМА УНО ХОЛЬМБЕРГА 1911 И 1913 ГГ (Voyages chez les Oudmourtes et les Maris. Lettres d'Uno Holmberg 1911 et 1913), под РЕДАКЦИЕЙ С. ЛАЛЛУККИ, Т.Г. МИННИЯХМЕТОВОЙ, Р.Р. САДИКОВА, СЕРИЯ «ФИНЛЯНДИЯ В РОССИИ», САНКТ- ПЕТЕРБУРГ: ИЗДАТЕЛЬСТВО «ЕВРОПЕЙСКИЙ ДОМ, 224 С. 
SADIKOV, 2008 = САДИКОВ РАНУС РАФИКОВИЧ, ТРАДИЦИОННЫЕ РЕЛИГИОЗНЫЕ ВЕРОВАНИЯ И ОБРЯДНОСТЬ ЗАКАМСКИХ УДМУРТОВ (ИСТОРИЯ И СОВРЕМЕННЫЕ ТЕНДЕНЦИИ РАЗВИТИЯ) (Mes croyances traditionnelles religieuses et les rituels des Oudmourtes d'outre-Kama - histoire et tendances actuelles), УФА: ЦЕНТР ЭТНОЛОГИЧЕСКИХ ИССЛЕДОВАНИЙ УНЦ РАН, 232 С.

SALMIN, 2007 = САЛМИН АНТОН КИРИЛЛОВИЧ, СИСТЕМА ФОЛЬК-РЕЛИГИИ ЧУВАШЕЙ (Le système de la religion populaire des Tchouvaches), САНКТ-ПЕТЕРБУРГ: НАУКА, 605 С.

SEMENOV, 1992 = СЕМЕНОВ ВИКТОР АНАТОЛЬЕВИЧ, ТРАДИЦИОННАЯ СЕМЕЙНАЯ ОБРЯДНОСТЬ НАРОДОВ ЕВРОПЕЙСКОГО СЕВЕРА: К РЕКОНСТРУКЦИИ МИФОЛОГИЧЕСКИХ ПРЕДСТАВЛЕНИЙ КОМИ (ЗЫРЯН) (Les rituels familiaux traditionnels des peuples du Nord européen : reconstruction des représentations mythologiques des Komis zyriènes), САНКТ-ПЕТЕРБУРГ, 152 C.

SITNIKOV, 2006 = СИТНИКОВ КОНСТАНТИН ИВАНОВИЧ, СЛОВАРЬ МАРИЙСКОЙ МИФОЛОГИИ. ТОМ 1. БОГИ, ДУХИ, ГЕРОИ (Dictionnaire de la mythologie marie. Tome 1 : Dieux, esprits, héros), ЙОШКАР-ОЛА, $160 \mathrm{C}$.

SMIRNOV, 1890 = СМИРНОВ ИВАН НИКОЛАЕВИЧ, Н., ВОТЯКИ (Les Votiaks), ИОАИЭ, Т. VIII, ВЫП. 2. КАЗАНЬ, 308 C.

STEINGOLD, 2006 = ШТЕЙНГОЛЬД АНЖЕЛИКА, ОТРАЖЕНИЕ ДРЕВНЕСЛАВЯНСКИХ ВЕРОВАНИЙ В РУССКОМ ЛЕксиконЕ (Reflets des croyances des anciens Slaves dans le lexique russe), Tartu Ülikooli Kirjastus, 202 C.

ŠUTOVA, 2001 = ШУТОВА НАТАЛЬЯ ИВАНОВНА, ДОХРИСТИАНСКИЕ КУЛЬТОВЫЕ ПАМЯТНИКИ В УДМУРТСКОЙ РЕЛИГИОЗНОЙ ТРАДИЦИИ: ОПЫТ КОМПЛЕКСНОГО ИССЛЕДОВАНИЯ (Les monuments cultuels préchrétiens dans la tradition religieuse oudmourte. Une expérience de recherche complexe), ИЖЕВСК: УДМУРТСКИЙ ИНСТИТУТ ИСТОРИИ, ЯЗЫКА И ЛИТЕРАТУРЫ УРО РАН, 304 С.

TOJDYВEKOVA, 1997 = ТОЙДЫБЕКОВА ЛИДИЯ СЕМЁНОВНА, МАРИЙСКАЯ ЯЗЫЧЕСКАЯ ВЕРА И ЭТНИЧЕСКОЕ САМОСОЗНАНИЕ (La religion païenne marie et la conscience ethnique), Joensuu yliopistopaino, 397 C.

TOLSTAJA, 2010 = ТОЛСТАЯ СВЕТЛАНА МИХАЙЛОВНА, СЕМАНТИЧЕСКИЕ КАТЕГОРИИ ЯЗЫКА КУЛЬТУРЫ: ОЧЕРКИ ПО СЛАВЯНСКОЙ ЭТНОЛИНГВИСТИКЕ (Catégories sémantiques du langage de la culture : essais d'ethnolinguistique slave), москвА: книжный дом «лиьроком», 368 с.

USAČEVA, 2008 = УСАЧЕВА ВАЛЕРИЯ ВАСИЛЬЕВНА, МАГИЯ СЛОВА И ДЕЙСТВИЯ В НАРОДНОЙ КУЛЬТУРЕ СЛАВЯН (La magie de la parole et de l'action dans la culture populaire des Slaves), МОсКВА: ИНСТИТУТ СЛАВЯНОВЕДЕНИЯ РАН, 368 С.

VEREŠČAGIN, 1995 = ВЕРЕЩАГИН ГРИГОРИЙ ЕГОРОВИЧ, ВОТЯКИ СОСНОВСКОГО КРАЯ (Les Votiaks du kraj de Sosnovka), СОБР. СОч. В 6 ТОМАХ. Т. І., ИЖЕВСК: УИИЯЛ УРО РАН, 260 С.

VLADYKINA, 2006 = ВЛАДЫКИНА ТАТЬЯНА ГРИГОРЬЕВНА, «АТРИБУТИВНЫЕ И ВЕРБАЛЬНЫЕ ПАРАЛЛЕЛИ В УДМУРТСКОМ И РУССКОМ СВАДЕБНОМ РИТУАЛАХ» (Parallèles attributifs et verbaux dans les rituels nuptiaux oudmourtes et russes), РУССКИЙ СЕВЕР И ВОСТОЧНЫЕ ФИННО-УГРЫ: ПРОБЛЕМЫ ПРОСТРАНСТВЕННО-ВРЕМЕННОГО ФОЛЬКЛОРНОГО ДИАЛОГА (Le Nord russe et les Finno-Ougriens orientaux : problèmes d'un dialogue des oralités dans l'espace et dans le temps): МАТЕРИАЛЫ I МЕЖРЕГИОНАЛЬНОЙ КОНФЕРЕНЦИИ И VII МЕЖДУНАРОДНОЙ ШКОЛЫ МОЛОДОГО ФОЛЬКЛОРИСТА. ИЖЕВСК, 23-26 ОКТЯБРЯ 2005 Г. / ОТВ. РЕД. В.М. ГАЦАК, Т.Г. ВЛАДЫКИНА, ИЖЕВСК: АНК, сс. 50-57.

Travaux de terrain de l'auteur (=TTA) 
Raïon ДE Kijasovo, République d'Oudmourtie

Dubrovskij

1. Anisimova Galina Anatol'evna (1970)

2. Bespalova Antonida Petrovna (1950)

3. Nikonova Marija Pavlovna (1926)

Staraja Sal'ja

1. Kulikova Zinaida Francova (1954)

2. Kulikov Arkadij Nikolaevič (1948)

3. Pavlova Galina Nikolaevna (1957)

4. Petuhova Raisa Ivanovna (1956)

Raïon de Malopurga, République d'Oudmourtie Malaja Purga

1. Karabaeva Marija Ivanovna (1961)

Raïon de Možga, République d'Oudmourtie Bol'blique

1. Aleksandrova Elizaveta Sergeevna (1931)

2. Rekalova Nina Nikolaevna (1938)

Starye Kaksi

1. Širšina Evdokija Ivanovna (1931)

Iževsk, République d'Oudmourtie

1. Čybyševa Fljura Ahmethanovna (1962)

Raïon de Tatyšly, République du Baškortostan

Novye Tatyšly

1. Gil'manova Gašira Šajmardanovna (1933)

Urazgul'dy

1. Mentdijarova Fljura Menkairovna (1951)

2. Nurtdinova Madina Šajhutdinovna (1955)

3. Rijanova Zoja Menkairovna (1964)

Raïon дE Vavož, République d'Oudmourtie

Gurez'-Pudga

1. Ščetkina Antonida Petrovna (1939-)

\section{Matériaux d'archives}

NII et GGPI, 1987 = ЗАПИСИ ФОЛЬКЛОРНОЙ ЭКСПЕДИЦИИ НИИ И ГГПИ, 15 ДЕКАБРЯ 1987 Г.,

Д. УДМУРТ КАРАУЛ КРАСНОГОРСКОГО РАЙОНА УАССР. ЗАПИСАЛИ ХОДЫРЕВА М.Г., КАРПОВА Л.Л.

\section{NOTES}

1. Cette variante de la traduction littérale est proposée par T.G. Vladykina et G. A. Gluhova (2011, p. 121).

2. «Le village des morts» désigne le cimetière. On trouve des désignations analogues du cimetière, par exemple, chez les Russes (СЕлениЕ мЕРтвых, «le village des morts", погост, le cimetière du village), les Mordves (ТонАчИнь ВЕлЕ, « le village qui se trouve de l'autre côté »), et les Ougriens de l'Ob' (ХАЛАС ПУГ'ОР, « le bourg des morts ») (Petruhin, 1995, p. 314 ; Zen'ko, 1997, p. 109 ; Karabel'nikov, ressource électronique).

3. Le coin de l'isba dans lequel se trouvent les icônes (N.d.T.). 
4. Les traductions sont de l'auteur. (N.d.T. : les traductions sont faites à partir de la traduction en russe de l'auteur).

5. Dans la culture populaire oudmourte, le lexique vulgaire russe est un des moyens de parler d'une manière détournée du contact avec l'autre monde, de même que la prière païenne pendant les prières, les commémorations et autres.

6. Cette information a été reçue pendant la rédaction de l'article. La correspondante a souhaité garder l'anonymat.

7. Pendant la présentation orale de V. Semenova, doctorante à l'université de Tartu, née en 1987, district de Kušmar, raïon de Zvenigovskij, République du Mari El. Cette information a été obtenue pendant la rédaction de l'article.

8. Un élément de construction des toits, la partie la plus haute, ayant souvent la forme d'un cheval (N.d.T.).

9. En confirmant l'appellation de ce personnage démonologique, R.R. Sadikov remarque que «chez les musulmans l'ange de la mort s'appelle Azrail » (Sadikov, 2008, p. 154). Il est possible qu'à ce personnage soit lié un autre mot oudmourte, эзЕль (du tatare әЖәл, « moment de la mort, mort, décès »), qui signifie la mort, le décès, le sort, le destin. L'aire de distribution de ce terme est plus large que celui d'Azrali, et sans doute a-t-il été bien plus anciennement assimilé chez les Oudmourtes.

10. En mari : АЗЫРЕHE.

11. En tchouvache : чун илли.

12. En tchouvache : ЭСРЕЛ.

13. Voir en détail Sadikov, 2008, p. 154 ; Sitnikov, 2006, p. 17.

14. D'autres attributs du meurtre en faisaient partie: la corde, la faux, la hache, l'alêne, le marteau en bois, la pelle, la serpe (voir les détails chez Sitnikov, 2006, p. 17).

15. Des représentations semblables sont caractéristiques, par exemple, des Komis. Selon leurs croyances, les ancêtres morts leur apportent leur protection, «apparaissant devant ceux qu'ils protègent dans les situations difficiles » (Mifologija komi, 1999, p. 96).

16. Pil'mon (russe Filimon) désigne dans ce contexte le nom de la famille à laquelle appartient Fedor, le protagoniste du récit oral.

\section{RÉSUMÉS}

En se fondant sur des matériaux publiés et sur ses travaux de terrain, l'auteur étudie les récits oraux et les croyances des Oudmourtes qui concernent les représentations de l'état précédant la mort d'un individu, de sa mort, et de sa « visite » temporaire dans l'autre monde. Il examine les variantes locales de termes oudmourtes liés aux phénomènes d'avant-mort. L'accent est mis principalement sur les stéréotypes comportementaux de la communication, dont la sémantique est analysée à travers le prisme de figures symboliques, d'actions et d'unités verbales.

On the basis of published materials and of the author's fieldwork, this article studies oral narratives and beliefs of the Udmurt about the one's pre-death state, one's death and the provisional stay in the world. It presents also the local forms of Udmurt terms about these states. The article insists on behavioural communication stereotypes, whose semantics is studied through the prism of symbolic metaphors, actions and verbal units. 
Tuginedes publitseeritud materjalidele ja välitöödele uurib autor udmurtide suulisi jutustusi ning uskumusi, mis puudutavad isiku surmale eelnevat seisu, tema surma ja tema ajutist „külastust” teispoolsusse. Artiklis uuritakse kohalikke variante udmurdi sõnavarast, mis on seotud surmaeelsete nähtustega. Põhirõhk on asetatud kommunikatsiooni käitumisstereotüüpidele, mille semantikat analüüsitakse sümboolsete kujundite, tegevuste ja sõnaliste üksuste vaatevinklist.

НА ОСНОВЕ ОПУБЛИКОВАННЫХ МАТЕРИАЛОВ И ПОЛЕВЫХ СВЕДЕНИЙ АВТОРА ИЗУЧАЮТСЯ УСТНЫЕ РАССКАЗЫ И ПОВЕРЬЯ УДМУРТОВ О ПРЕДСТАВЛЕНИЯХ ПРЕДСМЕРТНОГО СОСТОЯНИИ ЧЕЛОВЕКА, СМЕРТИ И ВРЕМЕННО «ПОБЫВАВШЕГО» В ИНОМ МИРЕ. ПРИВОДЯТСЯ ЛОКАЛЬНЫЕ ВАРИАНТЫ УДМУРТСКИХ ТЕРМИНОВ, СВЯЗАННЫХ С ОКОЛОСМЕРТНЫМИ ЯВЛЕНИЯМИ. ОСНОВНОЙ АКЦЕНТ СДЕЛАН НА ПОВЕДЕНЧЕСКИХ СТЕРЕОТИПАХ КОММУНИКАЦИИ, СЕМАНТИКА КОТОРОЙ РАССМАТРИВАЕТСЯ СКВОЗЬ ПРИЗМУ СИМВОЛИЧЕСКИХ ОБРАЗОВ, ДЕЙСТВИЙ И ВЕРБАЛЬНЫХ Единиц.

\section{INDEX}

Mots-clés : agent/esprit de la mort, agent/esprit de la mort, ancêtres défunts, ancêtres défunts, communication, communication, décès, décès, liminalité, liminalité, monde des morts, monde des morts, monde des vivants, monde des vivants, Oudmourtes, Oudmourtes, représentations d'avant-mort, représentations d'avant-mort, signes de la mort, signes de la mort, « visite » de l'autre monde, « visite » de l'autre monde

nomsmotscles Besermans Khakasses, Komis permiaks, Maris, Mordves, Oudmourtes, Ougriens de l'Ob, Tchouvaches

motscleset elavate maailm, kommunikatsioon, liminaalsus, representatsioonid enne surma, surmavaim, surm, surma märgid, surnud esivanemad, surnute maailm, teise ilma külastus, udmurdid

Index chronologique : XXIe siècle (début)

Index géographique : Bol’šie Siby, Debëssy Dubrovskij, Gurez'-Pudga, Inde, Iževsk, Kiasovo, Kušmar, Malaja Purga, Možga, Novye Tatyšly, République du Mari El, République d'Oudmourtie, République du Baškortostan, Staraja Sal'ja, Starye Kaksi, Tatyšly, Udmurt Karaul, Ujvaj, Urazgil'dy, Zvenigovskij (raïon)

motsclesru АГЕНТ/ДУХ СМЕРТИ, ЗНАКИ СМЕРТИ, КОММУНИКАТИВНОЕ ПОВЕДЕНИЕ, КОНЧИНА, ЛИМИНАЛЬНОСТЬ, МИР ЖИВЫХ, МИР МЕРТВЫХ, ОКОЛОСМЕРТНЫЕ ПРЕДСТАВЛЕНИЯ, ПОКОЙНЫЕ ПРЕДКИ, «ПОСЕЩЕНИЕ» ИНОГО МИРА, УДМУРТЫ

Keywords : agent of death, communication, dead ancestors, death, death signs, liminality, predeath representations, Udmurt, "visit" in the other world, world of the dead, world of the living disciplines oudmourte, russe, tatare

Thèmes : études de folklore, ethnologie 